\title{
Quality of Life in Patients With Primary and Metastatic Brain Tumors in the Literature as Assessed by the FACT-Br
}

\author{
Nicholas Chiu ${ }^{\mathrm{a}}$, Leonard Chiu ${ }^{\mathrm{a}}$, Liang Zeng ${ }^{\mathrm{a}}$, Liying Zhang ${ }^{\mathrm{a}}$, David Cella ${ }^{\mathrm{b}}$, Marko Popovic ${ }^{\mathrm{a}}$, \\ Ronald Chow ${ }^{\mathrm{a}}$, Henry Lam ${ }^{\mathrm{a}}$, Michael Poon ${ }^{\mathrm{a}}$, Edward Chow ${ }^{\mathrm{a}, \mathrm{c}}$
}

\begin{abstract}
Background: The Functional Assessment of Cancer TherapyBrain (FACT-Br) is a quality of life (QOL) assessment tool that was originally developed for use in patients with primary brain tumors. However, the tool has also been used to assess QOL in patients with metastatic brain tumors. The purpose of this study is to compare the differences in QOL responses as assessed by the FACT-Br in patients with primary and metastatic brain neoplasms.
\end{abstract}

Methods: A systematic literature search was conducted using the OvidSP platform in MEDLINE (1946 to July Week 2 2012) and EMBASE (1980 to 2012 Week 28). Articles in which the FACT-Br was used as a QOL assessment for patients with malignant brain tumors (both primary and metastatic) were included in the study. The weighted means of FACT-Br subscale and overall scores were calculated for the studies. To compare these scores, weighted analysis of variance was conducted and PROC GLM was performed for the data. A P-value of $<0.05$ was considered statistically significant.

Results: A total of 23 studies (four in brain metastases, 18 in primary brain tumors and 1 in a mixed sample) using the FACT-Br for assessment of QOL were identified. Social and functional well-being were significantly better in patients with primary brain tumors (weighted mean score of 22.2 vs. $10.7, \mathrm{P}=0.0026,16.9$ vs. $6.2, \mathrm{P}$ $=0.0025$, respectively). No other scale of the FACT-Br was significantly different between the two groups and the performance status of patients included in both groups was similar.

Conclusions: Patients with primary brain cancer seemed to have better social and functional well-being scores than those with meta-

Manuscript accepted for publication December 11, 2012

${ }^{a}$ Rapid Response Radiotherapy Program, Department of Radiation Oncology, Odette Cancer Centre, Sunnybrook Health Sciences Centre, University of Toronto, Toronto, Ontario, Canada

${ }^{b}$ Department of Medical Social Sciences, Northwestern University

Feinberg School of Medicine, Chicago, IL, USA

${ }^{\mathrm{c} C o r r e s p o n d i n g ~ a u t h o r: ~ E d w a r d ~ C h o w, ~ D e p a r t m e n t ~ o f ~ R a d i a t i o n ~}$ Oncology, Odette Cancer Centre, Sunnybrook Health Sciences Centre, 2075 Bayview Avenue, Toronto, ON, M4N 3M5, Canada.

Email: Edward.Chow@sunnybrook.ca

doi: http://dx.doi.org/10.4021/wjon585w static brain tumors. Other QOL domains were similar between these two groups. However, the heterogeneity in the included studies and the low sample size of included samples in patients with metastatic brain tumors could have confounded our findings.

Keywords: FACT-Br; Quality of Life; Brain metastases; Primary brain tumors

\section{Introduction}

Malignant brain tumors can be broadly divided into primary brain tumors (namely tumours originating in the brain) and secondary brain tumors (namely brain metastases). Both cause significant morbidity for affected individuals. These patients typically have very short life expectancies: in metastatic brain tumor patients, the median survival has been found to be within several months [1]; in primary brain tumor patients, median survival is not much better, typically within a few months or years [2]. Patients with metastatic brain tumors also face burden from other possible systemic diseases. As the prognosis is extremely poor, palliation, rather than cure, is often the more suitable treatment for this patient population. In many cases, management and prevention of complications are the targets of treatment. As such, quality of life (QOL) is an important consideration.

QOL is a subjective, multidimensional construct that focuses on several key domains that emphasize a patient's wellbeing [3]. As QOL reflects a patient's individual situation, it is most commonly assessed through the use of selfreported questionnaires completed by the patient.

The Functional Assessment of Cancer Therapy (FACT) group offers questionnaires developed for the assessment of QOL in cancer patients. In addition to the FACT-General (FACT-G), a core questionnaire used to determine the more general domains of QOL among all cancer patients, other disease-specific questionnaires are also available. The FACT-Brain (FACT-Br) is one such instrument that assesses brain-tumor related QOL issues. This tool consists of the FACT-G plus a brain-tumor specific scale. A total of 50 items are included that cover the following domains of QOL: phys- 
ical well-being, social/family well-being, emotional wellbeing, functional well-being, and disease specific concerns. Patients are asked to indicate the presence/severity of certain issues/symptoms on a scale of 0 - 4 (a 5-point Likert Scale).

While the FACT-Br was originally developed for use in patients with primary brain tumors, the tool has also been used to assess QOL in patients with metastatic brain tumors. How patients with primary brain tumors and patients with metastatic tumors respond to the FACT-Br may be of interest to health-care professionals in understanding the potential differences between QOL in the two patient populations.

The purpose of this study is to compare and evaluate the difference in FACT-Br QOL responses between patients with primary brain tumors and metastatic brain tumors as reported in the literature.

\section{Methods and Materials}

A systematic literature search was conducted over the OvidSP platform in MEDLINE (1946 to July Week 2 2012) and EMBASE (1980 to 2012 Week 28). The following search terms were used in a variety of combinations: FACT-Br, FACT-Brain, Functional Assessment of Cancer Therapy, FACT, primary tumor, brain metastases, palliative, quality of life, questionnaire, and instrument assessment. No restrictions were made on language.

Articles in which the FACT-Br was used as a QOL assessment for patients with malignant brain tumors (both primary and metastatic) were included in the study. Studies were only included if prospective data from the FACT-Br were available. Primary outcomes of interest were FACTBr scores and demographic parameters of patients included in the studies (namely gender, primary cancers, median age, etc.). Although articles in which patients' baseline subscale scores for the FACT-Br were of primary interest, articles that only included the overall FACT-Br score were also included in the study. Reference lists of extracted studies were explored along with lists of other publications that cited the reference. Articles were identified and data were extracted independently by five authors.

The scores of the FACT-Br and demographic data were extracted for all included studies. To compare the median KPS and average FACT-Br scales in patients with primary and metastatic brain tumors, weighted analysis of variance (ANOVA), based on the number of patients in the QOL data reported, was conducted and PROC GLM was performed for the data. To normalize the distribution of KPS and FACT-Br scales, natural log-transformation was applied. The weighted arithmetic means and the weighted standard deviation (SD) of the FACT-Br were also calculated in patients with primary and metastatic brain tumors. The weighted mean was defined as in Figure 1, and the weighted variance was defined as in Figure 2, where $\mathrm{w}_{i}$ is the weight for the $i$ th strudy, $\mathrm{x}_{i}$ is the

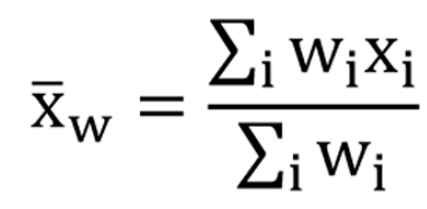

Figure 1. The weighted mean.

$$
\mathrm{s}_{\mathrm{w}}^{2}=\frac{1}{\mathrm{~d}} \sum_{\mathrm{i}} \mathrm{w}_{\mathrm{i}}\left(\mathrm{x}_{\mathrm{i}}-\overline{\mathrm{x}}_{\mathrm{w}}\right)^{2}
$$

Figure 2. The weighted variance.

$i$ th variable value, and the divisor $d$ is $n-1$. The weighted variance is a measure of variability, and it is the sum of the weighted squared distance of data values from the mean divided by the variance divisor which is defined to be $n-1$. A P-value of $<0.05$ was considered statistical significant. All analyses were conducted using Statistical Analysis of Software (SAS version 9.2 for Windows).

\section{Results}

A total of 635 publications were identified in the search. Of those, a total of 23 studies using the FACT-Br for assessment of QOL in patients with malignant brain tumors were identified with reported QOL results [2, 4-25]. Of the 23 studies (29 arms in total), 4 studies utilized the FACT-Br as an assessment tool for QOL in patients with brain metastases [4, 12, 18, 24]; one of the identified studies used the FACT-Br for both primary and metastatic patients [13]; and the remaining 18 studies composed of patients with primary brain cancer[2, 5-11, 14-17, 19-23, 25].

The FACT-Br was published in 1995 by Michael A. Weitzner et al [20]. All the studies using FACT-Br were published afterwards with sample sizes ranging from 7 to 718 . The included studies came from medical centers across 5 different countries: 16 publications from the United States [6-9, 11-20, 22, 23], 3 from Canada [2, 4, 25], 2 from Italy [5, 10], and 1 each from China [24] and Japan [21]. Butler et al and Roger et al $[13,18]$ were excluded from analysis as the former included patients with both primary and metastatic brain tumors and the latter only reported the median FACT$\mathrm{Br}$ score, rather than the mean.

\section{Demographics and clinical characteristics}

While the majority of the studies included did not disclose whether their patients were in-patients or out-patients, 4 of the identified studies indicated that their patient sample was entirely composed of in-patients $[5,11,17,19]$ only one 


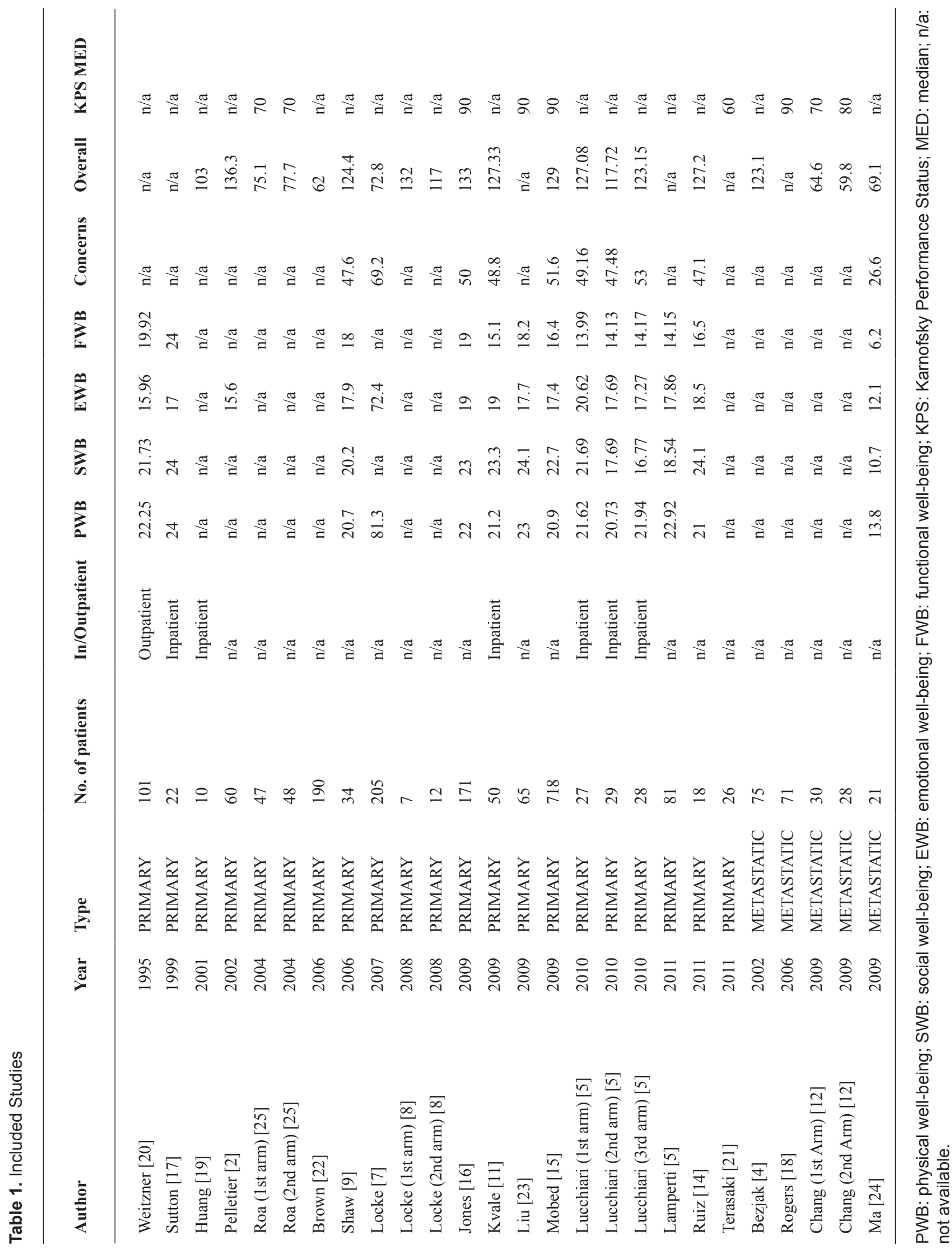


Table 2. Weighted Means of KPS and FACT-Br Subscale Scores

\begin{tabular}{|c|c|c|c|c|c|}
\hline & \multicolumn{2}{|c|}{ Primary Brain Tumors } & \multicolumn{2}{|c|}{ Metastatic Brain Tumor } & \multirow{2}{*}{ P-value } \\
\hline & $\begin{array}{l}\text { Sum of } \\
\text { Weight }\end{array}$ & Weighted Mean (SD) & $\begin{array}{l}\text { Sum of } \\
\text { Weight }\end{array}$ & $\begin{array}{l}\text { Weighted Mean } \\
\text { (SD) }\end{array}$ & \\
\hline KPS & 1,075 & $87.51(104.61)$ & 129 & $83.18(66.32)$ & 0.6631 \\
\hline Physical well-being & 1,549 & $29.38(230.56)$ & 21 & $13.8\left(\mathrm{NA}^{*}\right)$ & 0.5952 \\
\hline Social well-being & 1,344 & $22.23(17.54)$ & 21 & 10.7 (NA) & 0.0026 \\
\hline Emotional well-being & 1,609 & $24.58(203.53)$ & 21 & 12.10 (NA) & 0.6412 \\
\hline Functional well-being & 1,344 & $16.92(20.34)$ & 21 & 6.20 (NA) & 0.0025 \\
\hline $\begin{array}{l}\text { Brain subscale: Concerns related to } \\
\text { Brain Metastasis }\end{array}$ & 1,280 & $53.81(86.37)$ & 21 & 26.60 (NA) & 0.0619 \\
\hline Overall Scale & 1,654 & $111.27(290.24)$ & 154 & $92.83(212.13)$ & 0.4386 \\
\hline
\end{tabular}

* NA: not available because there was only one study with available subscales; $\uparrow$ : P-value was obtained by weighted analysis of variance; natural log-transformation was applied for KPS, FACT-Br subscales, and overall scale.

study explicitly indicated an exclusively out-patient sample (Table 1) [20].

Of the 29 study arms included in the review, 5 included a sample composed of $40.0 \%$ to $49.9 \%$ male patients [ $4-6$, $12,24], 11$ included a sample composed of $50.0 \%$ to $59.9 \%$ male patients $[2,5,8,9,11,13,15,20,25], 11$ included a sample composed of $60.0 \%$ to $69.9 \%$ [5, 7, 21-26, 27], and 2 included a sample composed of 70.0-79.9\% male patients $[10,17]$.

Mean age was reported in 18 of 29 of the arms while median age was reported in 16 (some reported both). The majority of studies included patients equal to or greater than 40 years of mean age $[2,5-8,10,11,15,16,19,20,22,25]$. In studies that reported the median age, all included patients equal to or greater than 40 years of age $[5,7-9,12-15,18$, 21-23].

\section{Weighted means}

The Median KPS and average FACT-Br scales are shown in Table 1. Weighted means and P-values were calculated for KPS and FACT-Br scales in patients from primary or metastatic brain tumor groups (Table 2).

For primary brain tumor patients, the weighted mean of their median KPS scores was 87.51; in comparison, for metastatic brain tumor patients, the weighted mean of their median KPS scores was 83.18. The difference was not statistically significant $(\mathrm{P}=0.66)$.

Unfortunately, while 3 included studies (totalling a com- bined sample size of 154 patients) provided overall FACT-G scores $[4,12,24]$, only one of them included subscale score data for the FACT-Br [24].

The mean overall FACT-Br (FACT-G + Br Subscale) score in patients with primary brain tumors was $111.27[2,5$, $7,8,11,14-16,19,22,25]$. In comparison, the mean overall FACT-Br score in patients with metastatic brain tumors was $92.83[4,12,24]$. However, this difference was not statistically significant $(\mathrm{P}=0.44)$. For the brain subscale, the mean subscale score in patients with primary brain tumors was $53.81[9,11,14,15]$ whereas that in patients with metastatic brain tumors was 26.60 [24]. Again, this difference was not statistically significant $(\mathrm{P}=0.062)$ (Table 2$)$.

Patients with primary brain tumors generally had worse QOL as assessed by the FACT-G component. Physical wellbeing, social/family well-being, emotional well-being, and functional well-being for primary vs. metastatic patients were: 29.38 vs. $13.60,22.23$ vs. $10.70,24.58$ vs. 12.10 , and 16.92 vs. 6.20 respectively.

There was a statistical difference in the social and functional well-being scales between patients with primary and metastatic brain tumors $(\mathrm{P}=0.0026$, and $\mathrm{P}=0.0025)$ (Table 2 ). This suggests that patients with metastatic brain tumors were more likely to have a statistically significant worsening in social and functional well-being subscale scores compared to those with primary brain tumors. There was no statistical significant difference in median KPS and other FACT$\mathrm{Br}$ subscales between patients with primary and metastatic brain tumors. 


\section{Discussion}

A trend was noticed in the differences between metastatic patient responses vs. primary patient responses on the FACTBr. Metastatic patient response scores on the FACT-Br seemed to be lower on all 4 wellbeing subscales as well as the additional brain subscale and the overall FACT-Br score - seemingly suggesting a relatively better QOL for primary brain tumor patients. A statistical difference in the social and functional well-being scales between patients with primary and metastatic brain tumors was found.

To our knowledge, no studies have exclusively observed the difference in social and functional well-being in patients suffering from primary brain cancer compared to patients suffering from metastatic brain cancer. On the other hand, several studies have compared QOL between the two groups based on the stage of disease. Siddiqi et al investigated the difference in QOL between the primary and metastatic cancer patients in general [27]. They observed the differences in QOL (symptom severity and physical functioning) experienced by primary non-metastatic (PNM), primary metastatic (PM) and recurrent (RC) cancer patients. RC cancer patients reported the worst symptom severity and physical function followed by PM and PNM patients. These findings are consistent with our results, as the authors observed QOL to be generally worse in metastatic patients compared with primary patients: an observation that holds true for the current study as well.

An important limitation of our study is that only 5 studies used the FACT-Br as a QOL assessment tool in brain metastases patients; in addition, only one study actually included the individual subscale scores of the FACT-Br responses with a sample size of 21 patients. Thus the weighted average of individual subscale responses to the FACT-Br in metastatic brain tumor patients is solely based on patients' responses from this one study. With validation and continued adoption, future studies should continue to assess patients with brain metastases using the FACT-Br. KPS was not a confounding variable in this study because the difference in the weighted means of the median KPS scores obtained in the two patient populations was not found to be statistically significant.

Another limitation was the heterogeneity of the studies and patients included. Because the disease progression of patients in each study was not known entirely, progression could have varied greatly from patient to patient and from study to study. Such differences in disease progression could explain differences in QOL. For example, if brain tumor patients in all the primary cancer studies were further along in disease progression than the metastatic patients, a difference in QOL would, naturally, be expected.

The results of this study pave the way for possible courses of action in identifying more certainty in this field. Several recommendations for future research in the field include: first, more studies like the ones done by Chang et al [12],
Ma et al [24], and Bezjak et al [4] should be done using the FACT-Br as a QOL assessment tool in metastatic patients: as not many were found to exist in the databases included in the literature search. As more studies utilizing the FACT-Br in the metastatic patient population are conducted, the current study could be repeated to confirm the validity and accuracy of our results. More importantly, there is a need to validate the FACT-Br in the metastatic patient population.

\section{Conclusion}

Patients with primary brain cancer seem to have better social and functional well-being than those with metastatic brain tumors. Other QOL domains are similar between these two groups. The readers are cautioned of the limitations of our study.

\section{Acknowledgement}

We thank the generous support of Bratty Family Fund, Michael and Karyn Goldstein Cancer Research Fund, Joseph and Silvana Melara Cancer Research Fund, and Ofelia Cancer Research Fund.

\section{Conflicts of Interest}

The authors have no conflicts of interest to disclose.

\section{References}

1. Gaspar LE, Scott C, Murray K, Curran W. Validation of the RTOG recursive partitioning analysis (RPA) classification for brain metastases. Int J Radiat Oncol Biol Phys. 2000;47(4):1001-1006.

2. Pelletier G, Verhoef MJ, Khatri N, Hagen N. Quality of life in brain tumor patients: the relative contributions of depression, fatigue, emotional distress, and existential issues. J Neurooncol. 2002;57(1):41-49.

3. Chen E, Nguyen J, Zhang L, Zeng L, Holden L et al. Quality of life in patients with brain metastases using the EORTC QLQ-BN20 and QLQ-C30. Journal of Radiation Oncology. 2012; 1: 179-186.

4. Bezjak A, Adam J, Barton R, Panzarella T, Laperriere N, Wong CS, Mason W, et al. Symptom response after palliative radiotherapy for patients with brain metastases. Eur J Cancer. 2002;38(4):487-496.

5. Lucchiari C, Botturi A, Pravettoni G. The impact of decision models on self-perceived quality of life: a study on brain cancer patients. Ecancermedicalscience. 2010;4:187.

6. Munoz C, Juarez G, Munoz ML, Portnow J, Fineman I, 
Badie B, Mamelak A, et al. The quality of life of patients with malignant gliomas and their caregivers. Soc Work Health Care. 2008;47(4):455-478.

7. Locke DE, Decker PA, Sloan JA, Brown PD, Malec JF, Clark MM, Rummans TA, et al. Validation of singleitem linear analog scale assessment of quality of life in neuro-oncology patients. J Pain Symptom Manage. 2007;34(6):628-638.

8. Locke DE, Cerhan JH, Wu W, Malec JF, Clark MM, Rummans TA, Brown PD. Cognitive rehabilitation and problem-solving to improve quality of life of patients with primary brain tumors: a pilot study. J Support Oncol. 2008;6(8):383-391.

9. Shaw EG, Rosdhal R, D'Agostino RB, Jr., Lovato J, Naughton MJ, Robbins ME, Rapp SR. Phase II study of donepezil in irradiated brain tumor patients: effect on cognitive function, mood, and quality of life. J Clin Oncol. 2006;24(9):1415-1420.

10. Lamperti E, Pantaleo G, Finocchiaro CY, Silvani A, Botturi A, Gaviani P, Sarno L, et al. Recurrent brain tumour: the impact of illness on patient's life. Support Care Cancer. 2012;20(6):1327-1332.

11. Kvale EA, Murthy R, Taylor R, Lee JY, Nabors LB. Distress and quality of life in primary high-grade brain tumor patients. Support Care Cancer. 2009;17(7):793-799.

12. Chang EL, Wefel JS, Hess KR, Allen PK, Lang FF, Kornguth DG, Arbuckle RB, et al. Neurocognition in patients with brain metastases treated with radiosurgery or radiosurgery plus whole-brain irradiation: a randomised controlled trial. Lancet Oncol. 2009;10(11):1037-1044.

13. Butler JM, Jr., Case LD, Atkins J, Frizzell B, Sanders G, Griffin P, Lesser G, et al. A phase III, double-blind, placebo-controlled prospective randomized clinical trial of d-threo-methylphenidate $\mathrm{HCl}$ in brain tumor patients receiving radiation therapy. Int J Radiat Oncol Biol Phys. 2007;69(5):1496-1501.

14. Ruiz J, Case D, Enevold G, Rosdhal R, Tatter SB, Ellis TL, McQuellon RP, et al. A phase II trial of thalidomide and procarbazine in adult patients with recurrent or progressive malignant gliomas. J Neurooncol. 2012;106(3):611-617.

15. Mobed K, Liu R, Stewart S, Wrensch M, McCoy L et al. Quality of Life and Patterns of Use of Complementary and Alternative Medicines Among Glioma Patients. The Journal of Supportive Oncology. 2009; 7 (6): 23-31.

16. Jones LW, Cohen RR, Mabe SK, West MJ, Desjardins A, Vredenburgh JJ, Friedman AH, et al. Assessment of physical functioning in recurrent glioma: preliminary comparison of performance status to functional capacity testing. J Neurooncol. 2009;94(1):79-85.
17. Sutton LN, Radcliffe J, Goldwein JW, Phillips P, Janss AJ, Packer RJ, Zhao H. Quality of life of adult survivors of germinomas treated with craniospinal irradiation. Neurosurgery. 1999;45(6):1292-1297; discussion 12971298.

18. Rogers LR, Rock JP, Sills AK, Vogelbaum MA, Suh JH, Ellis TL, Stieber VW, et al. Results of a phase II trial of the GliaSite radiation therapy system for the treatment of newly diagnosed, resected single brain metastases. J Neurosurg. 2006;105(3):375-384.

19. Huang ME, Wartella JE, Kreutzer JS. Functional outcomes and quality of life in patients with brain tumors: a preliminary report. Arch Phys Med Rehabil. 2001;82(11):1540-1546.

20. Weitzner MA, Meyers CA, Gelke CK, Byrne KS, Cella DF, Levin VA. The Functional Assessment of Cancer Therapy (FACT) scale. Development of a brain subscale and revalidation of the general version (FACTG) in patients with primary brain tumors. Cancer. 1995;75(5):1151-1161.

21. Terasaki M, Eto T, Nakashima S, Okada Y, Ogo E, Sugita Y, Tokutomi T, et al. A pilot study of hypofractionated radiation therapy with temozolomide for adults with glioblastoma multiforme. J Neurooncol. 2011;102(2):247253.

22. Brown PD, Ballman KV, Rummans TA, Maurer MJ, Sloan JA, Boeve BF, Gupta L, et al. Prospective study of quality of life in adults with newly diagnosed high-grade gliomas. J Neurooncol. 2006;76(3):283-291.

23. Liu R, Solheim K, Polley MY, Lamborn KR, Page M, Fedoroff A, Rabbitt J, et al. Quality of life in low-grade glioma patients receiving temozolomide. Neuro Oncol. 2009;11(1):59-68.

24. Ma S, Xu Y, Deng Q, Yu X. Treatment of brain metastasis from non-small cell lung cancer with whole brain radiotherapy and Gefitinib in a Chinese population. Lung Cancer. 2009;65(2):198-203.

25. Roa W, Brasher PM, Bauman G, Anthes M, Bruera E, Chan A, Fisher B, et al. Abbreviated course of radiation therapy in older patients with glioblastoma multiforme: a prospective randomized clinical trial. J Clin Oncol. 2004;22(9):1583-1588.

26. Lien K, Zeng L, Nguyen J, Cramarossa G, Cella D, Chang E, Caissie A, et al. FACT-Br for assessment of quality of life in patients receiving treatment for brain metastases: a literature review. Expert Rev Pharmacoecon Outcomes Res. 2011;11(6):701-708.

27. Siddiqi A, Given CW, Given B, Sikorskii A. Quality of life among patients with primary, metastatic and recurrent cancer. Eur J Cancer Care (Engl). 2009;18(1):84-96. 